\title{
SUPERCRITICAL STABILITY OF AN AXIALLY MOVING BEAM PART I: MODEL AND EQUILIBRIUM ANALYSIS
}

\author{
S.-J. Hwang And N. C. Perkins \\ Mechanical Engineering and Applied Mechanics, The University of Michigan. \\ Ann Arbor, Michigan 48109, U.S.A.
}

(Received 29 March 1990 and in final form 1 March 1991)

\begin{abstract}
Axially moving material problems consider the dynamic response, vibration and stability of long, slender members which are in a state of translation. This study focuses on the response of axially moving beam-like elements at translation speeds that exceed the classical "critical speed stability limit". A non-linear model for an axially moving beam is derived that accounts for the initial beam curvature induced by supporting pulleys or wheels. Presently, the model is used to determine steady responses at critical and supercritical translation speeds. The properties of the equilibrium problem are examined using an approximate linear solution and an exact, non-linear solution. The deficiency of the linear solution is illustrated by its inability to capture essential features of the equilibrium problem particularly near and above the critical speed. In this high-speed region, the translating beam undergoes large overall buckling deformations leading to multiple and bifurcated equilibrium states. The stability of the equilibria is assessed in Part II.
\end{abstract}

\section{INTRODUCTION}

Many mechanical systems utilize a slender, translating element as a means of transmitting power, information and material. The term axially moving materials [1] collectively refers to the translating elements found in diverse applications including magnetic tape recording devices, belt and chain drives, thread and fiber winders, band saws, paper and web handling machinery, overhead conveyer systems and cable pay-out/reel-in systems. Recent developments in the literature on axially moving materials are reviewed in reference [2].

Models for the dynamics of axially moving materials consider the transverse response of the element as it translates with prescribed speed between supporting wheels, pulleys or guides. During operation, the element is subjected to tension forces which originate from the support and drive mechanisms. Traditionally, the tension is assumed to be sufficiently large so that the element is, initially, drawn perfectly straight. The analysis of such geometrically perfect elements leads to the following important conclusions regarding vibration and stability.

For flexible materials (e.g., chains, fibers, tapes and threads) the element is modeled as a taut string with negligible flexural rigidity $[3,4]$. For this model, the linear equation for free, transverse motion is a one-dimensional wave equation for a moving medium [5], and admits a d'Alembert solution involving two component waves with distinct propagation speeds in the downstream and upstream directions. When the translation speed equals the sound speed, the component wave propagating in the upstream direction becomes stationary and standing wave resonance leads to divergence instability. This instability occurs simultaneously for all free vibration modes. Thus, stable operations are limited to translation speeds below a theoretical critical speed which, in the case of flexible materials. is simply the sound speed. 
A critical speed also limits the stability of translating elements that possess flexural rigidity (e.g., bands, belts, conveyor webs and paper) which are modeled as translating beams [6-8] or plates [9]. However, in these cases separate critical speeds govern the stability of each vibration mode due to the dispersive nature of these fourth order models. In axially moving material applications, the boundaries are modeled as simple or clamped supports and stability is first lost by divergence. Depending on the degree of support compliance, however, the tension can increase with translation speed and result in a higher critical speed $[6,7]$. Free boundary conditions arise in the closely related system of a cantilevered pipe conveying a flowing fluid. In this case, the non-conservative fluid reaction forces at the free end of the pipe do work and stability is first lost by flutter. This latter problem has attracted considerable attention; see, for example, references [10] and [11].

The above conclusions are derived from models of highly tensioned, geometrically perfect axially moving materials. However, in applications, imperfections, such as initial curvature, arise which can never be fully eliminated within the practical limits imposed on system tension. Sources of initial curvature include bending moments generated by supporting wheels and pulleys, sag due to gravity, material imperfections and guide misalignment. Although often small in practice, these imperfections can play a dominant role in determining the system response.

For flexible materials (notably cables), any initial curvature due to sag leads to a stabilizing "speed-tensioning effect" $[12,13]$ which permits the sagged equilibrium to remain stable at speeds far above the "critical speed" predicted by the perfect (taut) string model. Also, for fiexible materials, a second equilibrium exists in which the element may stand vertically up in the shape of an arch. Both theory and experiment demonstrate that this arch-like equilibrium can be stabilized at sufficiently high translation speeds $[14,15]$. Small initial curvature also plays a key role in the analysis of coupled band/wheel systems $[16,17]$. In such systems, the bending moments and initial curvature generated at the band/wheel interface produce a coupling mechanism which leads to the transfer of vibration energy between spans.

The recent studies above demonstrate that neglecting even small imperfections may lead to serious errors in predicting the equilibrium, stability and dynamic response of axially moving materials. While the stability of flexible materials with imperfections is well understood, it is not known how imperfections alter the stability of systems which possess flexural rigidity. In particular, since the equilibrium of such fourth order systems is governed by a buckling-type stability problem $[6,18]$ the sensitivity to imperfections is potentially great.

The purpose of the present study is to investigate the stability of beam-like elements at speeds near to and above the classical "critical speed stability limit". The study focuses on the important effects of initial beam curvature that arises from the bending of the beam about two pulleys or wheels. In the first part of the paper, a theoretical model is derived that describes the non-linear, planar motion of a curved and translating beam. The model considers large, static beam deformations, and motion from equilibrium is described using a non-linear rod theory. The non-linear equations of equilibrium are extracted from the equations of motion and recast into a form which resembles the classical problem of the elastica. Exact equilibrium solutions, written in terms of elliptic integrals, are obtained to illustrate the steady response of the beam at both subcritical and supercritical translation speeds. Solution bifurcations occur near the critical speed, leading to multiple beam equilibrium states. The stability of these supercritical equilibrium states is described in detail in Part II [19].

\section{THEORETICAL MODEL}

A theoretical model is derived which governs the planar motion of a translating beam (e.g., band, belt, paper or web) with initial curvature. Figure 1 defines the problem of 


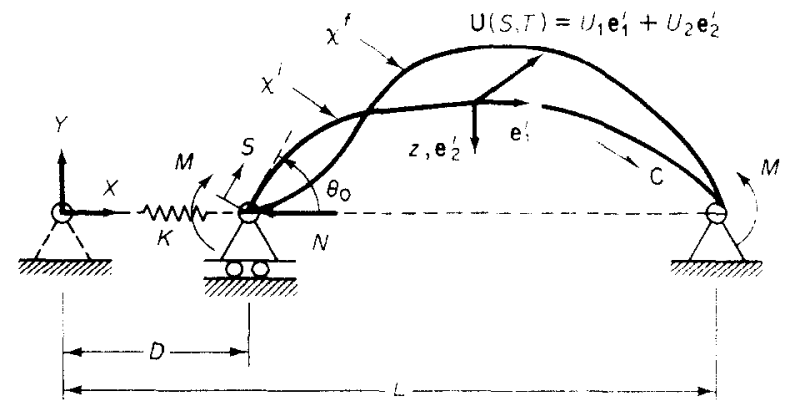

Figure 1. Definition diagram for a curved, axially moving beam. The beam centerline profile is shown in three states: (1) unbuckled configuration (dotted line), (2) buckled equilibrium configuration $\chi^{i}$, and (3) final configuration $\chi^{f}$. C denotes the particle translation velocity relative to $\chi^{\prime}$ and $\chi^{r}$

interest, and shows a beam which is translating between two supports and is initially held in equilibrium under tension $N$ and moments $M$ applied at the supports. The bending moments result from the curvature of the supporting wheels or pulleys (not shown) and the tension derives from an externally applied pre-load. Support flexibility is included in the model by allowing the left support to be elastically restrained in horizontal translation by a spring of stiffness $K$. The curve $\chi^{i}$ denotes the equilibrium configuration of the beam centerline profile which has length $L$ between the supports and lies in the $X-Y$ plane. The planar motion of the beam centerline profile about its equilibrium configuration is described by $\mathbf{U}(S, T)$, where $S$ denotes the arc length co-ordinate, measured along $\chi^{i}$, and $T$ denotes time. This motion, $\mathbf{U}(S, T)=U_{1} \mathbf{e}_{1}^{i}+U_{2} \mathbf{e}_{2}^{i}$, describes the final configuration of the centerline profile $\chi^{f}$, and is resolved into components aligned with the local tangential, $\mathbf{e}_{1}^{i}$, and normal, $\mathbf{e}_{2}^{i}$, directions defined by $\chi^{i}$. In Figure 1, $\mathbf{C}$ represents the beam particle translation velocity relative to the centerline profiles $\chi^{i}$ and $\chi^{f}$.

Hamilton's principle is used to derive the equations of motion which are based on the following assumptions: (1) the beam is a homogeneous, one-dimensional elastic continuum obeying a linear stress-strain relationship; (2) extensions of the beam are described by the Lagrangian strain of the centerline; (3) the motion of the beam is restricted to the $X-Y$ plane; (4) the beam may undergo large static deflections, and additional deflections from the curved equilibrium are described using a non-linear rod theory [20]; (5) rotary inertia due to bending and strain energy due to shear may be neglected assuming that the beam cross-sectional dimensions are small compared to its length; (6) gravitational and dissipative forces may be neglected; (7) the beam mass flux is constant. With these assumptions, expressions for strain energy, kinetic energy and work are derived for use in Hamilton's principle.

The strain energy of the beam is composed of terms associated with the extension of the beam centerline and flexure about the binormal (out-of-plane) axis. The Lagrangian strain $\varepsilon^{f}$ of the centerline in its final configuration $\chi^{f}$ is related to the displacements $U_{1}$ and $U_{2}$ and the curvature $\mathscr{K}^{i}$ of the centerline in its initial configuration $\chi^{i}$ through [13],

$$
\varepsilon^{\prime}=\varepsilon^{i}+\Delta \varepsilon
$$

where

$$
\Delta \varepsilon=U_{1 . S}-\mathscr{K}^{i} U_{2}+\frac{1}{2}\left[\left(U_{2, S}+\mathscr{K}^{i} U_{1}\right)^{2}+\left(U_{1, S}-\mathscr{K}^{i} U_{2}\right)^{2}\right]
$$

In these equations, ().s denotes partial differentiation with respect to $S$, and $\varepsilon^{\prime}$ is the Lagrangian strain of the beam centerline in $\chi^{i}$. In a similar fashion, the final curvature of 
the beam centerline $\mathscr{K}^{f}$ is related to the initial curvature $\mathscr{K}^{i}$ and the displacements $U_{\text {; }}$ and $U_{2}$ through

$$
\mathscr{K}^{f}=\mathscr{K}^{i}+\Delta \mathscr{K}
$$

where

$$
\Delta \mathscr{K}=a_{1} a_{2, S}, \quad a_{1}=1+U_{1, S}-\mathscr{K}^{i} U_{2}, \quad a_{2}=U_{2 . S}+\mathscr{K}^{i} U_{1} .
$$

The expression for $\Delta \mathscr{K}$ is derived from a non-linear extension of the rod theory developed in [20]. The strain energy of the beam in its final configuration $\chi^{f}$ is given by

$$
\pi_{s}^{f}=\int_{0}^{L} \int_{A} \frac{1}{2} E\left(\varepsilon^{f}-z \mathscr{K}^{f}\right)^{2} \mathrm{~d} A \mathrm{~d} S,
$$

where $z$ is a position co-ordinate in the normal direction measured from the beam centroidal axis (Figure 1), $E$ is Young's modulus, and $A$ is the beam cross-sectional area. Integration in equation (7) across the symmetrical beam area and use of equations (1) and (3) leads to

$$
\pi_{s}^{f}=\pi_{s}^{i}+\int_{0}^{L}\left[P^{i} \Delta \varepsilon+E I \mathscr{K}^{i} \Delta \mathscr{K}\right] \mathrm{d} S+\frac{1}{2} \int_{0}^{L}\left[E A(\Delta \varepsilon)^{2}+E I(\Delta \mathscr{K})^{2}\right] \mathrm{d} S,
$$

where $\pi_{s}^{i}$ is the strain energy of the curved beam in $\chi^{i}, P^{i}(S)=E A \varepsilon^{i}(S)$ is the beam tension in $\chi^{i}$ and $I$ is the principal area moment about the out-of-plane axis.

The kinetic energy of the curved beam in $\chi^{f}$ is given by

$$
\pi_{k}^{f}=\frac{1}{2} \int_{0}^{L}\left(\mathbf{V}^{f} * \mathbf{V}^{f}\right) \rho A \mathrm{~d} S
$$

where $\mathbf{V}^{f}$ represents the absolute velocity of a beam particle and $\rho$ is the beam density. The absolute velocity,

$$
\mathbf{V}^{f}=\left[U_{, T}+C U_{. S}\right]+C \mathbf{e}_{1}^{\prime},
$$

is the superposition of the velocity of $\chi^{f}$ and the velocity of the particle relative to $\chi^{f}$.

The work done by the bending moment $M$ and by the tension force $N$ is

$$
\begin{aligned}
\pi_{w}= & -\left.M\left(a_{2}+\theta\right)\right|_{0} ^{L}-N\left[U_{1}(0, T) \cos \theta_{0}+U_{2}(0, T) \sin \theta_{0}+D\right] \\
& +\frac{1}{2} K\left[U_{1}(0, T) \cos \theta_{0}+U_{2}(0, T) \sin \theta_{0}+D\right]^{2},
\end{aligned}
$$

where $\theta(S)$ is the angle of inclination of the beam centerline in $\chi^{i}, \theta_{0}=\theta(0)$, and $D$ is the horizontal distance the left support moves during the static deformation leading to $\chi^{i}$ (Figure 1). The distance $D$ is related to $\theta(S)$ through

$$
D=L-\int_{0}^{L} \cos \theta(S) \mathrm{d} S \text {. }
$$

Following Benjamin [10], the correct statement of Hamilton's principle for this system with mass transport is

$$
\delta\left[\int_{T_{1}}^{T_{2}}\left(\pi_{k}^{f}-\pi_{s}^{f}+\pi_{w}\right) \mathrm{d} T\right]-\left.\int_{T_{1}}^{T_{2}} \rho A C\left[\frac{\mathrm{d}}{\mathrm{d} T}\left(\mathbf{R}^{i}+\mathbf{U}\right)+C \mathbf{e}_{1}^{f}\right] * \delta \mathbf{U}\right|_{0} ^{L} \mathrm{~d} T=0 .
$$


Substituting equations (2)-(12) into equation (13) and taking the first variation leads to the following non-linear equations governing planar motion:

tangential component, $U_{1}$,

$$
\begin{gathered}
\left.\left[\left(P^{i}+A E \Delta \varepsilon\right) a_{1}\right]_{, s}-\left[\left(P^{i}+A E \Delta \varepsilon\right) \mathscr{K}^{i} a_{2}\right]-E I \mathscr{K}^{f} \mathscr{K}^{i}{ }_{. S} a_{1}+E I \mathscr{K}^{f}\left(a_{2, s}+\mathscr{K}^{i} a_{1}\right)\right]_{. s} \\
=\rho A\left\{\left(U_{1, T}+C a_{1}\right)_{, T}+C\left(U_{1, T}+C a_{1}\right)_{, s}-C \mathscr{K}^{i}\left(U_{2, T}+C a_{2}\right)\right\},
\end{gathered}
$$

normal component, $U_{2}$,

$$
\begin{gathered}
{\left[\left(P^{i}+A E \Delta \varepsilon\right) a_{2}\right]_{, s}+\mathscr{K}^{i} a_{1}\left[P^{i}+A E \Delta \varepsilon\right]+E I \mathscr{K}^{f} \mathscr{K}^{i} a_{2, s}-E I\left[\mathscr{K}^{f} a_{1}\right]_{. s s}} \\
=\rho A\left\{\left(U_{2, T}+C a_{2}\right)_{, T}+C\left(U_{2, T}+C a_{2}\right)_{. s}+C \mathscr{K}^{i}\left(U_{1, T}+C a_{1}\right)\right\},
\end{gathered}
$$

with the boundary terms:

$$
\begin{array}{cc}
{\left[\left(P^{i}+A E \Delta \varepsilon\right) a_{1}+E I \mathscr{K}^{f}\left(a_{2, S}+\mathscr{K}^{i} a_{1}\right)+M \mathscr{K}^{i}\right] \delta U_{1}=0} & \text { at } S=L, \\
\left\{\left(P^{\prime}+A E \Delta \varepsilon\right) a_{1}+E I \mathscr{K}^{f}\left(a_{2, S}+\mathscr{K}^{i} a_{1}\right)+M \mathscr{K}^{i}\right. & \\
\left.-\left[N+K\left(U_{1} \cos \theta_{0}+U_{2} \sin \theta_{0}+D\right)\right] \cos \theta_{0}\right\} \delta U_{1}=0 & \text { at } S=0, \\
{\left[\left(P^{i}+A E \Delta \varepsilon\right) a_{2}+E I\left(\mathscr{K}^{f} a_{2}\right)_{, S}\right] \delta U_{2}=0 \quad \text { at } S=L,} & \text { at } S=0, \\
\left\{\left(P^{i}+A E \Delta \varepsilon\right) a_{2}-E I\left(\mathscr{K}^{f} a_{1}\right)_{. S}\right. & \\
\left.-\left[N+K\left(U_{1} \cos \theta_{0}+U_{2} \sin \theta_{0}+D\right)\right] \sin \theta_{0}\right\} \delta U_{2}=0 & \text { at } S=0, L .
\end{array}
$$

The boundary conditions at the right support in Figure 1 are

$$
U_{1}=U_{2}=0 \quad \text { at } S=L, \quad E I \mathscr{K}^{f} a_{1}=-M \quad \text { at } S=L .
$$

At the left support, $\delta U_{1}$ and $\delta U_{2}$ must satisfy the geometric constraint $\delta U_{2}(0, T)=$ $\tan \theta_{0} \delta U_{1}(0, T)$ required for vanishing vertical support motion. Utilizing this constraint, the boundary conditions are:

$$
U_{2}=\tan \theta_{0} U_{1} \quad \text { at } S=0,
$$

$\left(P^{i}+A E \Delta \varepsilon\right)\left(a_{1} \cos \theta_{0}+a_{2} \sin \theta_{0}\right)-N-K\left(U_{1} \cos \theta_{0}+U_{2} \sin \theta_{0}+D\right)$

$$
\begin{gathered}
+M \mathscr{K}^{i} \cos \theta_{0}+E I \mathscr{K}^{f}\left(a_{2, s}+\mathscr{K}^{i} a_{1}\right) \cos \theta_{0}-E I\left(\mathscr{K}^{f} a_{1}\right)_{, s} \sin \theta_{0}=0 \quad \text { at } S=0, \\
E I \mathscr{K}^{f} a_{1}=-M \quad \text { at } S=0 .
\end{gathered}
$$

where equation (24) is a statement of horizontal force equilibrium.

For convenience in the subsequent analysis, the above model is non-dimensionalized using the following variable definitions:

$$
\begin{gathered}
\quad s=S / L, \quad \kappa=L \mathscr{K}^{i}, \quad u_{1}-U_{1} / L, \quad u_{2}=U_{2} / L, \quad d=D / L, \\
n=N L^{2} / E I, \quad m=M L / E I, \quad k=K L^{3} / E I, \quad \gamma=A L^{2} / I, \quad y=Y / L . \\
t=T \sqrt{E I / \rho A L^{2}}, \quad c^{2}=\rho A(L C)^{2} / E I, \quad p=P^{i} L^{2} / E I, \quad x=X / L .
\end{gathered}
$$

\section{EQUILIBRIUM AND BIFURCATION ANALYSIS}

The equations of equilibrium are extracted from the equations of motion (14) and (15) and the boundary conditions (21)-(25) by equating the displacement components $U_{1}$ and 
$U_{2}$ to zero. This procedure gives the following non-linear boundary-value problem for solution of the (non-dimensional) equilibrium beam curvature $\kappa(s)$ and tension $p(s)$ :

$$
p^{\prime}+\kappa \kappa^{\prime}=0, \quad 0<s<1, \quad \kappa^{\prime \prime}+\left(c^{2}-p\right) \kappa=0, \quad 0<s<1,
$$

with the boundary conditions

$$
\kappa(0)=\kappa(1)=-m, \quad p(0) \cos \theta_{0}-\kappa^{\prime}(0) \sin \theta_{0}=n+k d .
$$

The equilibrium of an axially moving beam was partially analyzed by Chubachi [6], whose linear analysis was used to predict the critical speeds of a simply supported belt that is initially drawn straight under large tension. Here, the complete non-linear equilibrium problem is presented, which leads to entirely new conclusions regarding the steady behavior of an axially moving beam. These new conclusions follow from the approximate (linear) analysis and the exact (non-linear) analysis described herein.

\subsection{APPROXIMATE ANALYSIS FOR SMALL CURVATURE}

As a first approximation, consider the case of small beam deflection and curvature for which the small angle approximations $\sin \theta \approx \theta$ and $\cos \theta \approx 1$ are valid. Under these conditions, the boundary-value problem takes the linearized form:

$$
\begin{gathered}
p^{\prime}=0, \quad 0<s<1, \quad \kappa^{\prime \prime}+\alpha^{2} \kappa=0, \quad 0<s<1, \\
\kappa(0)=\kappa(1)=-m, \quad p(0)=n,
\end{gathered}
$$

where the parameter $a=\sqrt{c^{2}-n}$ is a measure of the translation speed. Note that this problem admits the elementary solution:

$$
p(s)=n, \quad \kappa(s)=\frac{m}{1+\mathrm{e}^{\mathrm{i} \alpha}} \mathrm{e}^{\mathrm{i} \alpha s}+\frac{m}{1+\mathrm{e}^{-\mathrm{i} \alpha}} \mathrm{e}^{-\mathrm{i} \alpha s},
$$

provided that $\alpha$ is not an eigenvalue of the related homogeneous problem. If $\alpha=J \pi, J=$ $1,2, \ldots$, then neither the existence or uniqueness of a solution to the linear problem is guaranteed. These eigenvalues provide the critical speeds, $c=\sqrt{(J \pi)^{2}+n}$, which produce divergence instability (buckling) in the simply supported straight beams studied in [6-8]. The existence of the eigenvalues in the linear boundary-value problem suggests the existence of multiple solutions and bifurcations of solutions in the complete non-linear problem defined by equations (26)-(29).

\subsection{EXACT ANALYSIS FOR ARBITRARY CURVATURE}

The non-linear equilibrium problem is examined after recasting it into a form resembling the classical problem of the elastica [20]. Integrating equation (26) and substituting the result into equation (27) provides the uncoupled problem:

$$
\kappa^{\prime \prime}+\left(c^{2}-\beta+\frac{1}{2} \kappa^{2}\right) \kappa=0, \quad 0<s<1, \quad \kappa(0)=\kappa(1)=-m,
$$

where $\beta$ is a first integral of equation (26) given by

$$
\beta=p(s)+\frac{1}{2} \kappa^{2}(s)=c^{2} \sin \theta_{0}+(n+k d) \cos \theta_{0}+\frac{1}{2} m^{2} .
$$

This formulation of the equilibrium problem leads to the simple Duffing equation (35) and fundamental and higher order periodic solutions can readily be determined in terms of elliptic integrals. 
Integrating equation (35) leads to

$$
\mathrm{d} s= \pm \frac{\mathrm{d} \kappa}{\frac{1}{2} \sqrt{\left[\kappa^{2}-\kappa^{2}\left(s^{*}\right)\right]\left[4\left(\beta-c^{2}\right)-\kappa^{2}-\kappa^{2}\left(s^{*}\right)\right]}},
$$

where $s^{*}$ denotes a point at which $\kappa$ is an extremum. The extremum $\kappa\left(s^{*}\right)$ is determined from equation (37) after first computing $p\left(s^{*}\right)$ from a free body diagram of the beam between $s=0$ and $s=s^{*}$. At $s=s^{*}$, the tangent $\mathbf{e}_{1}^{i}$ is horizontal and the free body diagram provides

$$
p\left(s^{*}\right)=c^{2}\left(1-\cos \theta_{0}\right)+n+k d
$$

and therefore

$$
\kappa\left(s^{*}\right)= \pm \sqrt{4 \sin ^{2} \frac{\theta_{0}}{2}\left(c^{2} \cos \theta_{0}-n-k d\right)+m^{2}}
$$

Note that two unknown constants $\theta_{0}$ and $d$, which appear in equation (40), depend on the solution $\kappa(s)$. These constants and the solution must also satisfy the relation for $D$, equation (12), which (in non-dimensional form) provides

$$
1-d=\int_{0}^{1} \cos \theta(s) \mathrm{d} s
$$

The above integral can be evaluated from the solution $\kappa(s)$ using $\theta^{\prime}(s)=\kappa(s)$.

\subsubsection{Fundamental periodic solution}

The fundamental periodic solution corresponds to an equilibrium $\chi^{i}$ which resembles the fundamental buckling mode of a simply supported beam. This solution is symmetric about the mid-span, $s^{*}=\frac{1}{2}$.

Consider first the solution in the so-called "subcritical region" in which the translation speed is less than the first classical critical speed, $c \leqslant \sqrt{\pi^{2}+n}$. Using the change of variable

$$
\cos \eta=\sqrt{\frac{\kappa^{2}-\kappa^{2}\left(s^{*}\right)}{4\left(n+k d-c^{2} \cos \theta_{0}\right)}}
$$

in equation (38), and integrating provides the solution $\kappa(s)$ in terms of an incomplete elliptic integral of the first kind:

$$
\frac{1}{2}-s=\xi_{s u b} \int_{\phi_{s u b}(s)}^{\pi / 2} \frac{\mathrm{d} \eta}{ \pm \sqrt{1-\mu_{s u b}^{2} \sin ^{2} \eta}}, \quad 0 \leqslant s \leqslant s^{*}=\frac{1}{2}
$$

where

$$
\begin{gathered}
\xi_{s u b}=\frac{2}{\sqrt{4 \cos ^{2} \frac{\theta_{0}}{2}\left(n+k d-c^{2} \cos \theta_{0}\right)+m^{2}}} \\
\mu_{s u b}^{2}=\frac{4\left(n+k d-c^{2} \cos \theta_{0}\right)}{4 \cos ^{2} \frac{\theta_{0}}{2}\left(n+k d-c^{2} \cos \theta_{0}\right)+m^{2}}
\end{gathered}
$$




$$
\phi_{\text {sub }}(s)=\arcsin \sqrt{\frac{4 \cos ^{2} \frac{\theta_{0}}{2}\left(n+k d-c^{2} \cos \theta_{0}\right)+m^{2}-\kappa^{2}(s)}{4\left(n+k d-c^{2} \cos \theta_{0}\right)}}
$$

Note that application of the boundary condition $\kappa(0)=-m$ in equation (42) reduces it to a single non-linear equation in the two unknown constants $\theta_{0}$ and $d$ :

$$
\frac{1}{2}=\xi_{s u b} \int_{\phi_{s u b}(0)}^{\pi / 2} \frac{\mathrm{d} \eta}{ \pm \sqrt{1-\mu_{s u b}^{2} \sin ^{2} \eta}}, \quad 0 \leqslant s \leqslant s^{*}=\frac{1}{2} .
$$

Using equation (42) and the change of variable $2 \eta=\pi-\theta$ in equation (41) reduces equation (41) to a second equation in $\theta_{0}$ and $d$ :

$$
\frac{1-d}{2}=\xi_{s u b} \int_{\pi / 2-\theta_{0} / 2}^{\pi / 2} \frac{\left(1-2 \sin ^{2} \eta\right) \mathrm{d} \eta}{\sqrt{1-\mu_{s u b}^{2} \sin ^{2} \eta}} .
$$

The constants $\theta_{0}$ and $d$ are determined by simultaneous (numerical) solution of the nonlinear equations (46) and (47). Note that while four possible formulations of this result occur for the four combinations of signs in the expressions for $\kappa^{\prime}(s)$, equations (38) or (46), and $\kappa\left(s^{*}\right)$, equation (40), a solution exists only for the combination, $\kappa^{\prime}(s)>0$ on $0<s<s^{*}$ and $\kappa\left(s^{*}\right)<0$. For this solution, which is illustrated in section 4 , the angle $\theta_{0}>0$.

By contrast, multiple solutions are found in the "supercritical speed region" which is defined by $c>\sqrt{\pi^{2}+n}$. The first of these is found for the combination $\kappa^{\prime}(s)<0$ on $0<s<s^{*}$, equations (38) or (46), and $\kappa\left(s^{*}\right)<0$, equation (40). The change of variable

$$
\eta=\cos ^{-1} \frac{\kappa(s)}{-\sqrt{4 \sin ^{2} \frac{\theta_{0}}{2}\left(c^{2} \cos \theta_{0}-n-k d\right)+m^{2}}}
$$

in equation (38) leads to

$$
\frac{1}{2}-s=\xi_{\text {sup }} \int_{0}^{\phi_{\text {sup }}(s)} \frac{\mathrm{d} \eta}{\sqrt{1-\mu_{\text {sup }}^{2} \sin ^{2} \eta}}, \quad 0 \leqslant s \leqslant s^{*}=\frac{1}{2},
$$

where, in the supercritical speed region,

$$
\begin{gathered}
\xi_{\text {sup }}=\frac{1}{\sqrt{c^{2} \cos \theta_{0}-n-k d}}, \\
\mu_{\text {sup }}^{2}=\frac{4 \sin ^{2} \frac{\theta_{0}}{2}\left(c^{2} \cos \theta_{0}-n-k d\right)+m^{2}}{4\left(c^{2} \cos \theta_{0}-n-k d\right)}, \\
\phi_{\text {sup }}(s)=\arccos \frac{\kappa(s)}{-\sqrt{4 \sin ^{2} \frac{\theta_{0}}{2}\left(c^{2} \cos \theta_{0} \cdots n-k d\right)+m^{2}}} .
\end{gathered}
$$


Utilizing equation (48) in equation (41) with the change of variable

$$
\sin \eta=\frac{\sin \frac{\theta}{2}}{-\sqrt{4 \sin ^{2} \frac{\theta_{0}}{2}\left(c^{2} \cos \theta_{0}-n-k d\right)+m^{2}}}
$$

provides

$$
\frac{1-d}{2}=\xi_{\text {sup }} \int_{0}^{\phi_{\text {sup }}(0)} \frac{\left(1-2 \mu_{\text {sup }}^{2} \sin ^{2} \eta\right) \mathrm{d} \eta}{\sqrt{1-\mu_{\text {sup }}^{2} \sin ^{2} \eta}}
$$

As in the subcritical case, evaluation of equation (48) at the boundary condition $\kappa(0)=$ $-m$ leads to a single equation in $\theta_{0}$ and $d$ which is then solved numerically with equation (52). This solution provides a value for $\theta_{0}>0$, which is the continuous extension of the unique solution in the subcritical speed region.

In the supercritical speed region, a second (and possibly third) fundamental solution is found for the combination $\kappa^{\prime}(s)>0$ on $0<s<s^{*}$, equations (38) or (46), and $\kappa\left(s^{*}\right)>0$, equation (40). In this case, equation (38) leads to

$$
\frac{1}{2}-s=\xi_{\text {sup }} \int_{\phi_{\text {sup }}(s)}^{\pi} \frac{\mathrm{d} \eta}{\sqrt{1-\mu_{\text {sup }}^{2} \sin ^{2} \eta}}, \quad 0 \leqslant s \leqslant s^{*},
$$

and equation (41) leads to

$$
\frac{1-d}{2}=\xi_{\text {sup }} \int_{\phi_{\text {sup }}(0)}^{\pi} \frac{\left(1-2 \mu_{\text {sup }}^{2} \sin ^{2} \eta\right) \mathrm{d} \eta}{\sqrt{1-\mu_{\text {sup }}^{2} \sin ^{2} \eta}} .
$$

In deriving equations (53) and (54), the same changes of variables were employed as in the derivation of equations (48) and (52). Simultaneous solution of equations (53) (evaluated at $\kappa(0)=-m$ ) and (54) leads to either one or two solutions, depending on the magnitude of the translation speed $c$. For these latter solutions, $\theta_{0}<0$.

\subsubsection{Higher order periodic solutions}

In the supercritical speed region, a set of three higher order periodic solutions appears each time the translation speed approaches a higher order critical speed $c=\sqrt{(J \pi)^{2}+n}$, $J=2,3,4, \ldots$, associated with the straight beam model. For example, near $c=$ $\sqrt{(2 \pi)^{2}+n}$ a set of three $(J=2)$ equilibrium solutions appear which closely resemble the second (antisymmetric) buckling mode of a simply supported beam. Here, this set of solutions will be collectively referred to as the "second order solutions". Second and higher order solutions are computed using the same change of variables and parameters defined in section 3.2.1 for the supercritical speed region. For odd $J$, the solution for $\kappa(s)$ is described by

$$
\begin{aligned}
\frac{1}{2}-s= & \xi_{\text {sup }}\left[\frac{J \mp 1}{2} \int_{0}^{\pi} \frac{\mathrm{d} \eta}{\sqrt{1-\mu_{\text {sup }}^{2} \sin ^{2} \eta}}\right. \\
& \left. \pm \int_{0}^{\phi_{\text {sip }}(s)} \frac{\mathrm{d} \eta}{\sqrt{1-\mu_{\text {sup }}^{2}} \sin ^{2} \eta}\right], \quad 0 \leqslant s \leqslant s^{*},
\end{aligned}
$$


from which the definition for $d$, equation (41), provides

$$
\begin{aligned}
\frac{1-d}{2}= & \xi_{\text {sup }}\left[\frac{J \mp 1}{2} \int_{0}^{\pi} \frac{\left(1-2 \mu_{\text {sup }}^{2} \sin ^{2} \eta\right) \mathrm{d} \eta}{\sqrt{1-\mu_{\text {sup }}^{2} \sin ^{2} \eta}}\right. \\
& \left. \pm \int_{0}^{\phi_{\text {sup }}(0)} \frac{\left(1-2 \mu_{\text {sup }}^{2} \sin ^{2} \eta\right) \mathrm{d} \eta}{\sqrt{1-\mu_{\text {sup }}^{2} \sin ^{2} \eta}}\right] .
\end{aligned}
$$

For even $J$, these become

$$
\begin{aligned}
\frac{1}{2}-s= & \xi_{\text {sup }}\left[\frac{J}{2} \int_{0}^{\pi} \frac{\mathrm{d} \eta}{\sqrt{1-\mu_{\text {sup }}^{2} \sin ^{2} \eta}}\right. \\
& \left. \pm \int_{\phi(0)}^{\phi_{\text {sup }}(s)} \frac{\mathrm{d} \eta}{\sqrt{1-\mu_{\text {sup }}^{2} \sin ^{2} \eta}}\right], \quad 0 \leqslant s \leqslant s^{*},
\end{aligned}
$$

and

$$
\frac{1-d}{2}=\xi_{\text {sup }}\left[\frac{J}{2} \int_{0}^{\pi} \frac{\left(1-2 \mu_{\text {sup }}^{2} \sin ^{2} \eta\right) \mathrm{d} \eta}{\sqrt{1-\mu_{\text {sup }}^{2} \sin ^{2} \eta}}\right] .
$$

For a given solution order (i.e., specified value of $J$ ), the " $\mp$ " and " \pm " sign combinations appearing in equations (55)-(57) distinguish solutions having positive and negative values of $\theta_{0}$. For $\theta_{0}>0$, the upper sign in each pair is selected and a unique solution is always found. By contrast, for $\theta_{0}<0$, the lower sign in each pair is selected and either one or two solutions exist. The fundamental solution in the supercritical speed region, equation (48) with equation (52) and equation (53) with equation (54), are obtained from equations (55) and (56) for the special case $J=1$.

\section{RESULTS}

The axially moving beam equilibrium problem resembles an elastica buckling problem [20] wherein the translation speed plays the role of the buckling parameter [18]. This similarity is evident in Figure 2, in which it is shown how $\theta_{0}$, the angle of inclination of the beam centerline at the left support, depends on the translation speed. Results are shown for the fundamental, second and third order solutions for the simplest case of vanishing bending moment and support stiffncss $m=k=0$. The condition $m=0$ renders the equilibrium problem (35)-(36) homogeneous, and the trivial solution $\kappa(s)=\theta(s)=0$ is always a solution. In this case, the solution in the subcritical speed region, equations (42)-(47), provides only the trivial solution. Non-trivial solutions first appear as bifurcations from the trivial solutions at the first critical speed $c=\sqrt{\pi^{2}+n}$; see the solid curves in Figure 2. These non-trivial solutions represent the fundamental periodic solutions which satisfy equations (55) and (56) for $J=1$. Subsequent bifurcations of the trivial solution occur at higher order critical speeds and result in the non-trivial solutions represented by the dotted curves. These latter solutions represent the higher order solutions and they satisfy equations (57) and (58) for $J=2$ and equations (55) and (56) for $J=3$.

Multiple solutions also exist when an external bending moment is applied, as shown by the cases $m=0.25$ (solid curves) and $m=0.5$ (dashed curves) in Figure 3 . In such cases, the fundamental solution begins with the solution in the subcritical speed region, equations (42)-(47), which is continuous with the solution in the supercritical speed region given by equations (55) and (56) for $J=1$ and $\theta_{0}>0$. Also, in the supercritical speed region, two 
new fundamental periodic solutions appear slightly above the first classical speed, and they are computed as the multiple solutions of equations (55) and (56) for $J=1$ and $\theta_{0}<0$. The three branches of the fundamental solution are presently termed the "right-branch"

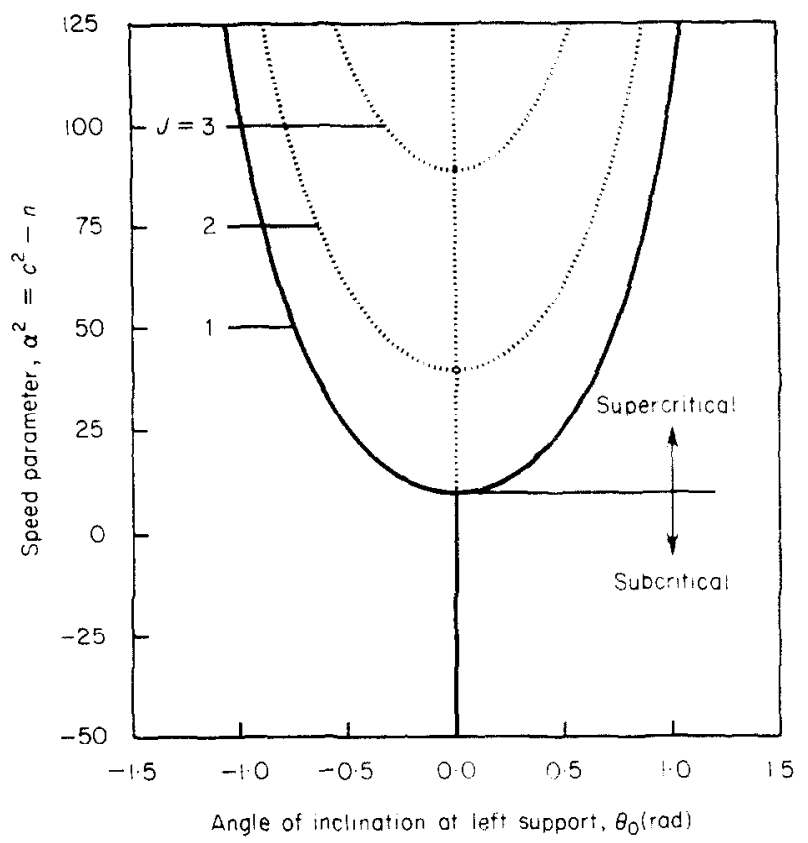

Figure 2. Bifurcations and multiple equilibria for the simplest case, $m=k=0$. Results are shown in the $a^{2} \theta_{0}$ plane for the case $n=100$. $J$ denotes the order of the solution and $\theta_{0}$ is the dependent variable.

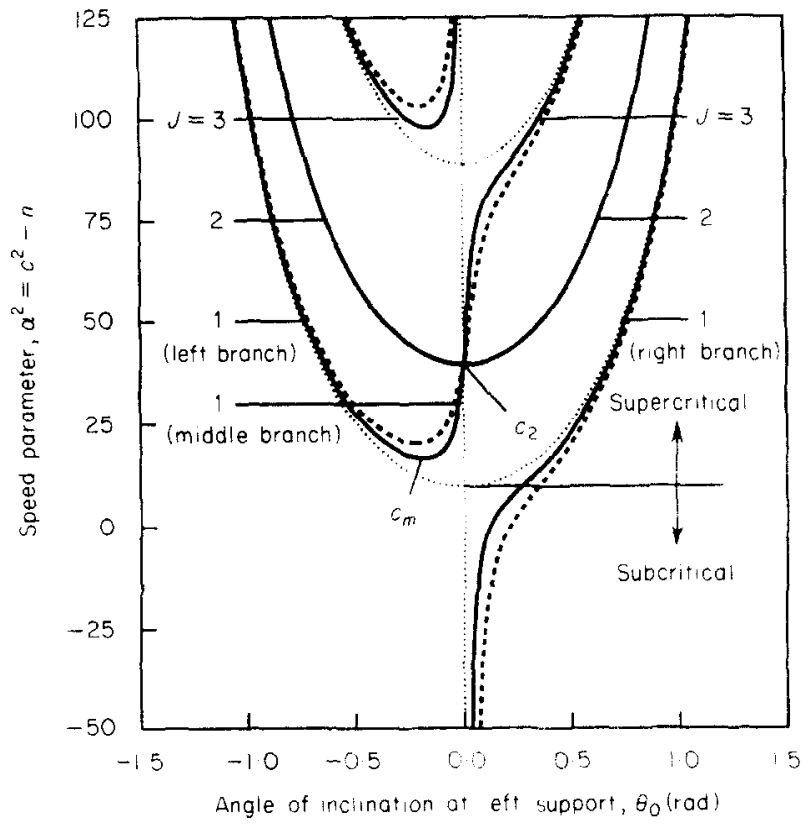

Figure 3. Multiple equilibria, bifurcations and unfoldings in the equilibrium problem. Results are shown in the $\alpha^{2}-\theta_{0}$ plane for the cases $m=0.5(\ldots), 0.25(\ldots)$ and $0(\cdots)$. In all cases $n=100$ and $k=0$. The index $J$ denotes the order of the solution and $\theta_{0}$ is the dependent variable. 
$\left(\theta_{0}>0\right)$, the "middle-branch" $\left(\theta_{0}<0\right.$ nearest trivial) and the "left-branch" $\left(\theta_{0}<0\right)$ equilibria, and are central to the stability analysis described in Part II of the paper. The previous solution corresponding to the case of an initially perfect beam $(m=0)$ is repeated for comparison; see the dotted curves. Note that the imperfection introduced by the bending moment $m$ leads to unfoldings of all the odd order bifurcations $(J=1,3, \ldots)$ seen in the case $m=0$ [21]. The odd order bifurcations are associated with symmetric buckling modes which are extremely sensitive to even slight bending moments. By contrast, the (symmetrically applied) bending moments have no influence on the antisymmetric buckling modes associated with the even order bifurcations $(J=2,4, \ldots)$. Consequently, the even order bifurcations do not unfold, and the solutions for $J=2,4, \ldots$ are independent of $m$. Note also that in the cases in which $m \neq 0$, the translation speed first required to produce multiple solutions, denoted $c_{m}$, is always greater than the fundamental classical critical speed and increases with increasing bending moment $m$. Note also that the middle-branch solution for $J=1$ is continuous with the right-branch solution for $J=3$ at the second critical speed denoted by $c_{2}$.

The actual shape of the equilibrium beam centerline is computed from the curvature solution $\kappa(s)$ by integrating the equations $\mathrm{d} x(s)=\cos \theta(s) \mathrm{d} s$ and $\mathrm{d} y(s)=\sin \theta(s) \mathrm{d} s$. Representative equilibria at subcritical and supercritical translation speeds for the case $m=$ 0.25 are shown in Figure 4. For this relatively small bending moment, the beam profile is nearly straight in the subcritical speed region and, for example, has a maximum deflection of only $0.3 \%$ of its length at $48 \%$ of the fundamental classical critical speed; see Figure 4(a). By contrast, the maximum beam deflections at speeds $110 \%$ and $143 \%$ of the critical speed are approximately $20 \%$ and $30 \%$ of the length, respectively; see Figures $4(\mathrm{~b})$ and

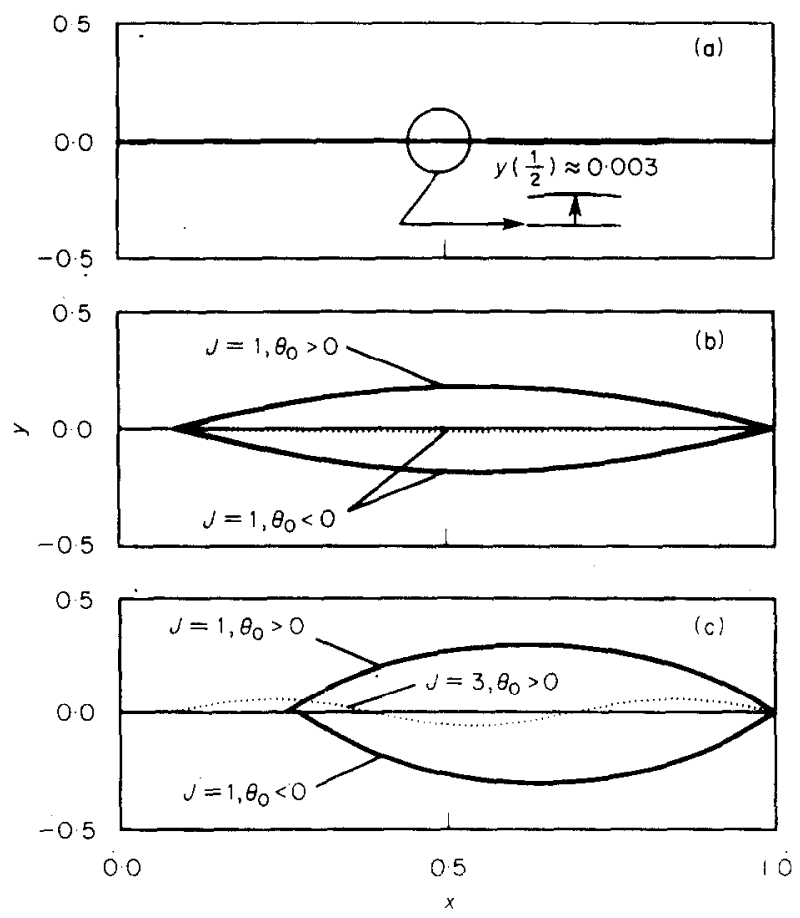

Figure 4. Representative equilibrium centerline profiles in the subcritical and supercritical speed regions. Equilibria shown for three translation speeds: (a) $c / \sqrt{\pi^{2}+n}=0.48$; (b) $c / \sqrt{\pi^{2}+n}=1 \cdot 10 ;$ (c) $c / \sqrt{\pi^{2}+n}=1 \cdot 43$. In all cases, $m=0 \cdot 25, n=100$ and $k=0$. J denotes the order of the solution. In the cases of multiple equilibria, the dotted curve denotes the equilibrium nearest to the trivial solution. 
(c). At $110 \%$ of the critical speed, the upper, central and lower profiles illustrated in Figure 4(b) correspond to the right, middle and left-branch equilibria $(J=1)$ in Figure 3 , respectively. At $143 \%$ of the critical speed, the translation speed exceeds the second classical critical speed. In this case, the middle profile in Figure 4(c), which again corresponds to a middle-branch in Figure 3, illustrates one form of the third order solution obtained using $J=3$ and $\theta_{0}>0$ in equations (55) and (56).

It is shown in Figure 4 that the equilibrium configuration for the fundamental solution becomes progressively more curved with increasing translation speed. This fact is, of course, already depicted in Figures 2 and 3 which show that $\left|\theta_{0}\right|$ approaches some large, limiting value as the translation speed approaches infinity. Note from equations (55) and (57) that, as the elliptic integrals are bounded, the factor $1 / \xi_{\text {sup }}$ must also remain bounded as $c \rightarrow \infty$. This factor remains bounded only if $\theta_{0} \rightarrow \pm \pi / 2$ as $c \rightarrow \infty$; refer to equation (49). Thus, unlike the classical elastica subjected to load-induced buckling, the ends of a translating beam can never rotate more than $90^{\circ}$ through speed-induced buckling.

The sensitivity of the equilibrium solution to support stiffness is illustrated in Figure 5 , which shows how $\theta_{0}$ depends on the translation speed for two extreme cases $k=0$ (solid curve), and $k=20000$ (dotted curve). In both cases $m=0 \cdot 25$ is used. Note that the speed required to produce multiple solutions, $c_{m}$, increases with $k$ as does the second order bifurcation speed, denoted $c_{2}$. As anticipated, a large support stiffness retards the formation of multiple equilibria.

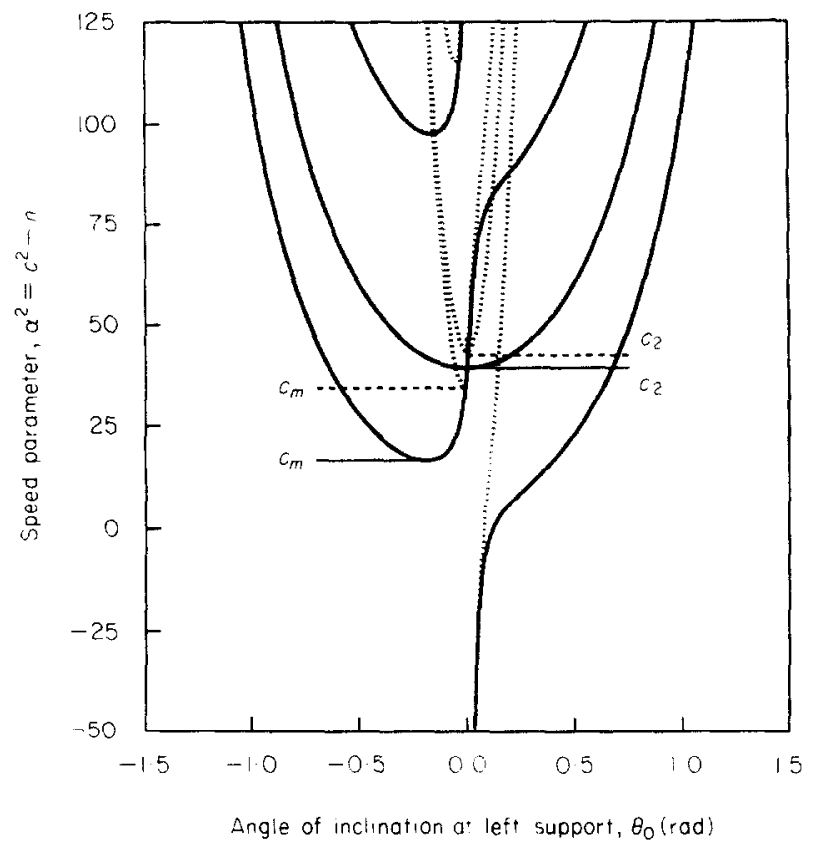

Figure 5. The sensitivity of the equilibrium solution to support stiffness. Results are shown in the $\alpha^{2}-\theta_{0}$ plane for the cases $k=0(\ldots)$ and $20000(\ldots)$. In both cases $m=0.25$ and $n=100$ and $\theta_{0}$ is the dependent variable.

It is clearly indicated in Figures 3 and 5 that the speed at which multiple equilibria first appear, $c_{m}$, depends on the applied bending moment $m$ and the support stiffness $k$. As described in Part II, the speed $c_{m}$ plays a key role in determining stability. Here, the dependence of $c_{m}$ on $m$ and $k$ is illustrated in Figure 6, which shows $c_{m}$ (solid curves) as a function of the bending moment $m$ for the cases $k=0$ and $k=20000$. In the special case of vanishing bending moment, $c_{m}$ is equal to the fundamental classical critical speed; refer 


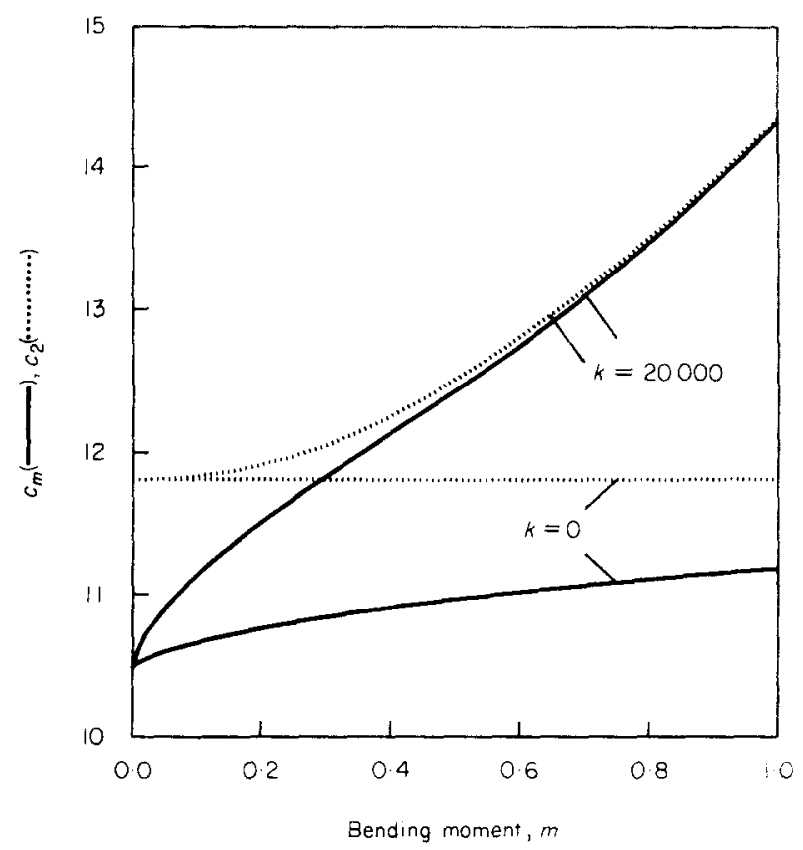

Figure 6. The sensitivity of $c_{m}$ and $c_{2}$ to bending moment and support stiffness. In both cases $n=100$ was used.

to Figure 2. As the bending moment increases, $c_{m}$ increases and asymptotically approaches from below the second order bifurcation speed, $c_{2}$, which is represented by the dotted curve.

An important comparison between the linear solution (section 3.1) and the non-linear solution (section 3.2) is made in order to illustrate the failure of the linear theory in the supercritical speed region. These solutions are depicted in Figure 7 for the case of $m=$ 0.25 and $k=0$. Note that in the subcritical speed region, the linear solution (dotted curve) represents a good approximation to the non-linear solution (solid curve). However, this approximation becomes quite poor near the first classical critical speed, where the linear solution becomes singular. In the supercritical speed region, the linear solution remains a reasonable approximation only to the non-linear solution which is closest to the trivial solution. In this region it cannot, of course, capture the many other non-trivial equilibria predicted by the non-linear solution.

\section{CONCLUSIONS}

A non-linear model for an axially moving beam is described which accounts for the initial curvature generated by supporting wheels and pulleys. The model considers geometrically non-linear beam deflections, and is therefore capable of describing systems operating at critical and supercritical translation speeds. The governing equations of equilibrium are presented and used to derive approximate (linear) and exact (non-linear) solutions for steady response. Both solutions indicate that the translating beam experiences large static deflections near the first critical speed and cannot remain straight. For the case of no applied bending moments, a bifurcation of the trivial solution occurs at the first critical speed and multiple equilibrium states are predicted from the non-linear analysis. This bifurcation unfolds when a slight bending moment is added, and the translation speed 


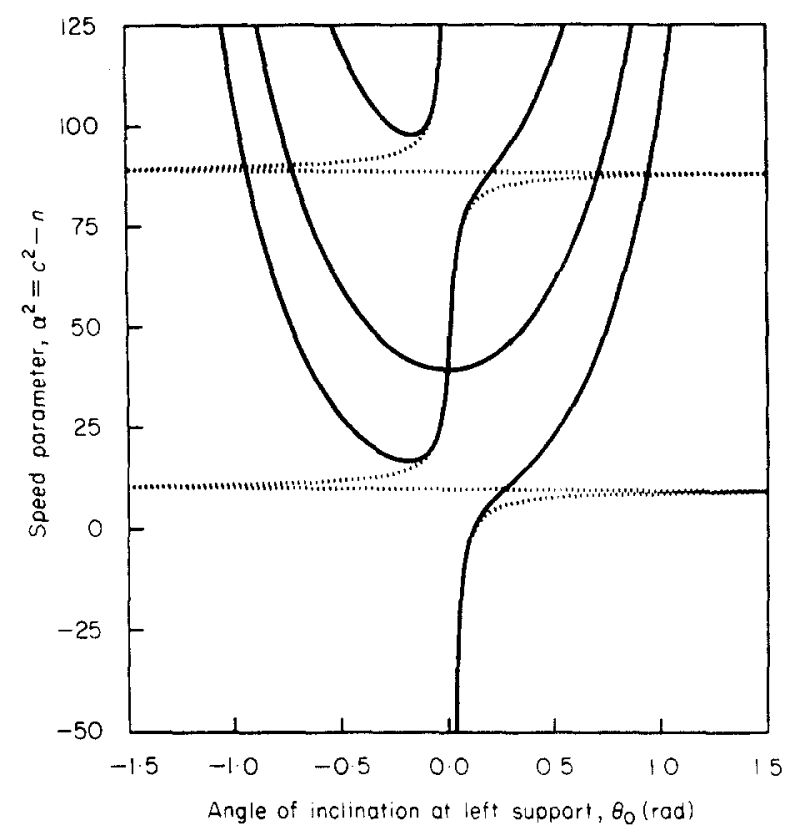

Figure 7. The failure of linear approximation. Linear $(\cdots \cdots)$ and non-linear $(-)$ solutions are shown in the subcritical and supercritical speed regions for the case $m=0 \cdot 25, n=100$ and $k=0$, with $\theta_{0}$ as the dependent variable.

required to first generate multiple equilibria increases. The stability of all supercritical equilibrium states is examined in detail in Part II [19].

\section{ACKNOWLEDGMENTS}

The authors gratefully acknowledge the support of the University of Michigan Office of the Vice President for Research, and the partial support of the U.S. Office of Naval Research Young Investigator Program.

\section{REFERENCES}

1. C. D. Mote, JR. 1972 Shock and Vibration Digest 4, 2-11. Dynamic stability of axially moving materials.

2. J. A. WICKeRT and C. D. Mote, JR. 1988 Shock and Vibration Digest 20, 3-13. Current research on the vibration and stability of axially moving materials.

3. R. A. SACK 1954 British Journal of Applied Physics 5, 224-226. Transverse oscillations in travelling strings.

4. S. Mahalingam 1957 British Journal of Applied Physics 8, 145-148. Transverse vibrations of power transmission chains.

5. W. L. MIRANKER 1960 IBM Journal of Research and Development 4, 36-42. The wave equation in a medium in motion.

5. T. Снuвachi 1957 Bulletin of the Japan Society of Mechanical Engineers 23, 205-210. Lateral vibration of axially moving wire or belt form materials.

7. C. D. Mote, JR. 1965 Journal of the Franklin Institute 279, 430-444. A study of bandsaw vibrations.

8. A. SimpSON 1973 Journal of Mechanical Engineering Science 15, 159-164. Transverse modes and frequencies of beams translating between fixed end supports.

9. A. G. UlsoY and C. D. Mote, J 1982 Journal of Engineering for Industry 104, 71-78. Vibration of wide band saw blades. 
10. T. B. Benjamin 1961 Proceedings of the Roval Society of London 261, series A, 457-499. Dynamics of a system of articulated pipes conveying fluid-Part I: Theory, and Part II Experiment.

11. R. W. Gregory and M. P. PAïdoussis 1966 Proceedings of the Royal Society of London 293, series A, 512-544. Unstable oscillation of tabular cantilevers conveying fluid Part $I$ : Theory, and Part II : Experiment.

12. A. Simpson 1972 Journal of Sound and Vibration 20, 177-189. On the oscillatory motions of translating elastic cables.

13. N. C. Perkins and C. D. Mote, JR. 1987 Journal of Sound and Vibration 114, 325-340. Threedimensional vibration of travelling elastic cables.

14. N. C. Perkins and C. D. Mote, JR. 1989 Journal of Sound and Vibration 128, 397-410. Theoretical and experimental stability of two translating cable equilibria.

15. N. C. PERKIns 1989 Journal of Sound and Vibration 135, 375-383. Asymptotic analysis of a translating cable arch.

16. K. W. WANG and C. D. Mote, JR. 1986 Journal of Sound and Vibration 109, 237-258. Vibration coupling analysis of band/wheel mechanical systems.

17. K. W. WANG and C. D. MorE, JR. 1987 Journal of Sound and Vibration 115, 203-216. Band/ wheel system vibration under impulsive boundary excitation.

18. J. M. T. Thompson and T. S. LuNN 1981 Journal of Sound and Vibration 77, 127-132. Static elastica formulations of a pipe conveying fluid.

19. S.-J. HWANG and N. C. PER KINS 1992 Journal of Sound and Vibration 154, 397-409. Supercritical stability of an axially moving beam, part II: vibration and stability analyses.

20. A. E. H. Love 1927 A Treatise on the Mathematical Theory of Elasticity. New York: Dover, 1944 republication of fourth edition. chapters 18,19 and 21.

21. M. Golubitsky, I. Stewart and D. G. SchaefFer 1987 Singularities and Groups in Bifurcation Theory. New York: Springer-Verlag. 